\title{
The ALMA view of the Antennae galaxy collision: How galaxy interaction triggers the formation of super star clusters
}

\author{
Cinthya N. Herrera ${ }^{1} \dagger$, François Boulanger ${ }^{2}$, Edith G. Falgarone ${ }^{3}$, \\ Guillaume Pineau des Forêts ${ }^{2}$, Santiago García-Burillo ${ }^{4}$, \\ Daisuke Iono ${ }^{1}$ and Pierre Guillard ${ }^{5}$ \\ ${ }^{1}$ National Astronomical Observatory of Japan, \\ 2-21-1 Osawa, Mitaka, Japan \\ email: herrera@iram.fr (present address), diono@nao.ac.jp \\ ${ }^{2}$ Institut d'Astrophyisque Spatiale \\ Université Paris Sud, Batiment 121, 91405 Orsay, France \\ email: francois.boulanger@ias.u-psud.fr, guillaume.pineaudesforets@ias.u-psud.fr \\ ${ }^{3}$ Ecole Normale Superieure, LERMA \\ 24 rue Lhomond, 75231 Paris, France \\ email: edith.falgarone@ens.fr \\ ${ }^{4}$ Observatorio Astronómico Nacional \\ Aptdo 1143, 28800 Alcalá de Henares, Spain \\ email: burillo@oan.es \\ ${ }^{5}$ Sorbonne Universités, UPMC Univ Paris 6 et CNRS, UMR 7095, \\ Institut d'Astrophysique de Paris 98 bis bd Arago, 75014 Paris, France \\ email: guillard@iap.fr
}

\begin{abstract}
The Antennae galaxies are a spectacular example of a burst of star formation triggered by the encounter of two galaxies, being an ideal source to understand how the dynamics of galaxy mergers drives star formation. We present archive ALMA CO(3-2) and VLT near-IR $\mathrm{H}_{2}$ spectro-imaging observations, and new ALMA ${ }^{13} \mathrm{CO}(2-1)$ and dust continuum observations, at $\sim 50$ pc resolution. Combining tracers of density and velocity structure of the gas and its energetics, we demonstrate that star formation involves a complex interplay of merger-driven gas dynamics and turbulence, and the dissipation of the gas kinetic energy. We focus on a compact, bright $\mathrm{H}_{2}$ source, associated with cold molecular gas and dust continuum emission, located where the velocity gradient in the interaction region is observed to be the largest. The characteristics of this source suggest that we are witnessing the formation, initiated by turbulent dissipation, of a cloud massive enough $\left(\sim 4 \times 10^{6} \mathrm{M}_{\odot}\right)$ to form a super star cluster within $1 \mathrm{Myr}$.
\end{abstract}

Keywords. ISM; galaxies: starbursts, interaction, star clusters

\section{Introduction}

Galaxy mergers and interactions are a common phenomenon in the Universe. They play a large role in determining the morphologies of galaxies and the properties of their stellar populations along the Hubble sequence. In mergers, most of massive star formation is observed in Super Star Clusters (SSCs), compact (few parsec size) and massive $\left(>10^{4} \mathrm{M}_{\odot}\right)$ star clusters. Our understanding on how the galaxy interaction triggers the formation of SSCs relies on theoretical arguments with only limited observational

$\dagger$ Present address: IRAM, 300 Rue de la Piscine, 38406 Saint Martin d'Hères, France. 
support. The formation of SSCs must involve a complex interplay of merger-driven gas dynamics, turbulence fed by the galaxy interaction, and dissipation of the kinetic energy of the gas. Hydrodynamical simulations suggest that massive complexes of cold gas, i.e. Super Giant Molecular Complexes (SGMCs), form where merger driven gas flows trigger compression, cooling and gravitational fragmentation (Teyssier et al. 2010). Within SGMCs, a hierarchy of structures must be present including pre-cluster clouds (PCCs), i.e. dense and compact concentrations of molecular gas that are sufficiently massive to form SSCs (Weidner et al. 2010).

We address this topic from an observer's standpoint, by studying the density and velocity structure of the gas and its energetics, which are essential observational information.

\section{Molecular gas in the Antennae overlap region}

The Antennae (at $22 \mathrm{Mpc}$ ) is a merger of two gas-rich spiral galaxies hosting thousands of SSCs (Whitmore et al. 1995). The most massive $\left(>10^{5} \mathrm{M}_{\odot}\right)$ and youngest $(<10 \mathrm{Myr})$ SSCs are located where the two disks interact - overlap region - and are associated with large amount of molecular gas fragmented into SGMCs (Wilson et al. 2000). Recent ALMA observations show that SGMCs have abundant substructures and complex velocity components (Herrera et al. 2012; hereafter H12; Whitmore et al. 2014). The large surface density of the CO molecular gas across the overlap region and its fragmentation into these massive clouds are evidence for cooling and gravitational fragmentation of the diffuse gas compressed in the galaxy collision (Herrera et al. 2011, hereafter H11).

Nature of the warm molecular gas emission. SINFONI/VLT observations revealed extended near-IR $\mathrm{H}_{2}$ emission associated with SGMCs (Fig. 1a). The cold (CO) and warm $\left(\mathrm{H}_{2}\right)$ molecular gas show similar morphologies and kinematics. Extinction-free diagnostics indicate that $\mathrm{H}_{2}$ is mainly excited by collisions. Most of the $\mathrm{H}_{2}$ emission traces the dissipation of the mechanical energy driven by the galaxy interaction. SGMCs would form through gas accretion and energy dissipation within converging flows driven by the galaxy interaction. The observed $\mathrm{H}_{2}$ luminosities of SGMCs can be accounted for by the loss of turbulent kinetic energy associated with the formation of gravitationally bound gas within convergent gas flows driven by the galaxy interaction (H11, H12).

Excitation of the cold molecular gas. ${ }^{13} \mathrm{CO}(2-1)$ observations obtained with ALMA during Cycle 2, at 0!' 4 angular resolution $(\sim 40 \mathrm{pc})$, trace the densest parts of the ${ }^{12} \mathrm{CO}$ molecular clouds (see Fig. 1 a). Fig. $1 \mathrm{~b}$ shows the $\mathrm{CO}(3-2) / \mathrm{CO}(2-1)$ ratio, a tracer of the gas excitation. The ratio changes between SGMCs, as seen in lower resolution observations (e.g., Ueda et al. 2012), and, as revealed now by ALMA, within individual SGMCs. In particular, SGMC 2 shows the largest variations. The gas can be heated by UV radiation (probably in SGMC 4/5 which has an embedded SSC, Herrera et al. 2015) and/or the dissipation of the gas turbulent kinetic energy.

In the overlap region, single dish observations measured a ${ }^{12} \mathrm{CO} /{ }^{13} \mathrm{CO}(2-1)$ ratio between 14 and 28 at $1.5 \mathrm{kpc}$ scales (Schulz et al. 2007), as also observed in other active star forming/interacting galaxies (e.g., Aalto et al. 1995). ALMA unveils ratios up to $\sim 50$ at 100 pc scales (Fig. 1b, towards PCC 1, see §3). Physical and chemical modelling of these ALMA data will be presented in a future paper. Different CO transitions and isotopes may have different filling factors at $100 \mathrm{pc}$ scales, and gas abundances of ${ }^{12} \mathrm{CO}$ and ${ }^{13} \mathrm{CO}$ may not be the canonical ones. For instance, towards the PCC 1 cloud, the $\mathrm{CO}(3-2) / \mathrm{CO}(2-1)$ ratio is 0.5 and ${ }^{12} \mathrm{CO} /{ }^{13} \mathrm{CO}$ ratio is $\sim 50$. 


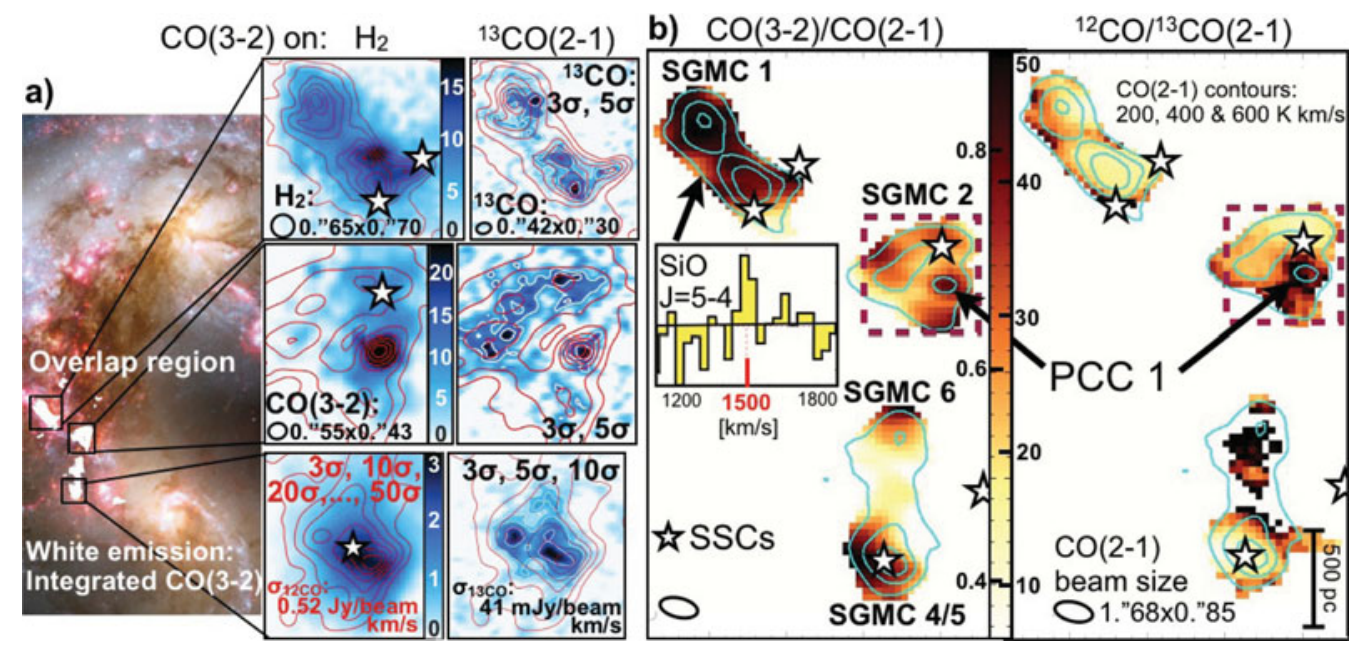

Figure 1. a) Integrated $\mathrm{CO}(3-2)$ emission from ALMA overlaid on an HST image of the Antennae. We zoom on the main SGMCs to show the distribution of the $\mathrm{H}_{2}, \mathrm{CO}(3-2)$ and ${ }^{13} \mathrm{CO}(2-1)$ integrated intensity lines. Red and white contours are the ${ }^{13} \mathrm{CO}(2-1)$ and $\mathrm{CO}(3-2)$ lines, respectively. $\mathrm{H}_{2}$ color bars are in units of $10^{-17} \mathrm{erg} \mathrm{s}^{-1} \mathrm{~cm}^{-1}$. b) $\mathrm{CO}$ integrated intensity ratios: $\mathrm{CO}(3-2)$ to $\mathrm{CO}(2-1)$ and ${ }^{12} \mathrm{CO}$ to ${ }^{13} \mathrm{CO}(2-1)$, respectively. Data were convolved to a common resolution, the $\mathrm{CO}(2-1)$ beam size. We highlight SGMC 2 and PCC 1 within it. The spectrum is the $\mathrm{SiO}$ profile of SGMC 1, after stacking each individual spectra to $1500 \mathrm{~km} \mathrm{~s}^{-1}$ based on the $\mathrm{H}_{2}$ velocity map. The most massive and youngest SSCs are marked with stars. ( $A$ color version of this figure is available online.)

The large variations of the CO line ratios reflect the complexity of physical processes in the Antennae overlap region. We are still missing more information of other $\mathrm{CO}$ transitions, specially its isotopes, to well constrain the gas properties at GMC scales.

\section{A pre-cluster cloud in the Antennae merger}

Our near-IR data obtained with the SINFONI spectro-imager on the VLT revealed a compact $\left(\sim 00^{\prime \prime} 6, \sim 50 \mathrm{pc}\right)$ source not associated with any known SSC (hereafter PCC 1; H11). With $\mathrm{CO}(3-2)$ ALMA observations we identified the CO counterpart of this PCC. It has the largest $\mathrm{H}_{2} / \mathrm{CO}$ emission ratio and coincides with the highest velocity shear in all of the overlap region (Fig. 2), i.e. the shortest dissipation timescale $\sim 1 \mathrm{Myr}$ (H12).

Herrera et al. (2011) proposed that the PCC 1 may be a massive cloud on its way to form a SSC within the next few Myr as its turbulent energy dissipates. Within this framework, the $\mathrm{H}_{2}$ lines trace turbulent energy that is being dissipated and radiated away, as the SGMC complexes and the PCC within, form. Based on dust continuum emission at $1.3 \mathrm{~mm}$ of our Cycle 2 ALMA observations, for PCC 1 we derive a mass of $\sim 4 \times 10^{6} \mathrm{M}_{\odot}$, which is one order of magnitude smaller than the virial mass estimated from the CO line-width and continuum size. Our mass estimate agrees with that obtained by Johnson et al. (2015) using the $870 \mu \mathrm{m}$ dust continuum emission. Johnson et al. argued that for the cloud to be confined it must be subject to a high $\left(\mathrm{P} / \mathrm{k}_{\mathrm{B}} \gtrsim 10^{8} \mathrm{~K} \mathrm{~cm}^{-3}\right)$ external pressure, which may be dominated by ram pressure.

Our ${ }^{13} \mathrm{CO}(2-1)$ and dust continuum emission observations do not resolve the internal structure of PCC 1. After beam deconvolution, we find $40 \pm 3$ and $49 \pm 6$ pc, respectively. The detection of non-resolved $\mathrm{HCO}+(4-3)$ and $\mathrm{HCN}(4-3)\left(n_{\text {crit }} \sim 10^{6}, 10^{7} \mathrm{~cm}^{-3}\right)$ 

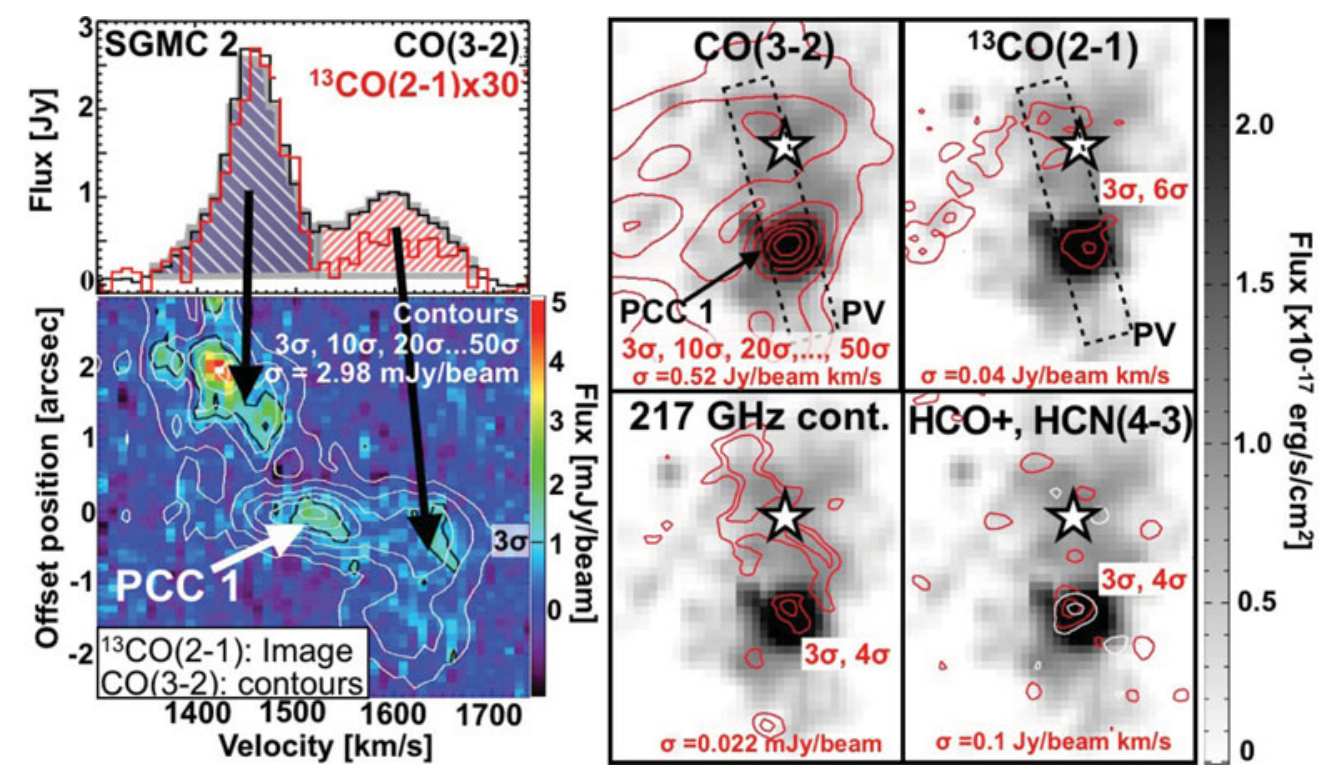

Figure 2. Top-left panel shows the total $\mathrm{CO}(3-2)$ and ${ }^{13} \mathrm{CO}(2-1)$ emission from SGMC 2. At the bottom, the position-velocity (PV) diagram across SGMC 2. PCC 1 is identified in CO and ${ }^{13} \mathrm{CO}$, and seems to be located at the interface of blue and red shifted gas. The right 4 panels show the $\mathrm{H}_{2}$ integrated emission in SGMC 2 in comparison to $\mathrm{CO}(3-2),{ }^{13} \mathrm{CO}(2-1), 1.3 \mathrm{~mm}$ continuum, $\mathrm{HCO}+(4-3)$ and $\mathrm{HCN}(4-3)$, in contours. PCC 1 is detected in all of these tracers. (A color version of this figure is available online.)

emission towards PCC 1 (Fig. 2) may indicate the presence of a gravitationally bound dense core within the source.

\section{References}

Aalto, S., Booth, R. S., Black, J. H., \& Johansson, L. E. B., 1995, A\&A, 300, 369

Flower, D. R. \& Pineau Des Forêts, G 2010, MNRAS, 406, 1745

Herrera, C. N., Boulanger, F., \& Nesvadba, N. P. H. 2011, A\& A, 534, A138

Herrera, C. N., Boulanger, F., Nesvadba, N. P. H., \& Falgarone, E. 2012, A\& A, 538, L9

Herrera, C. N \& Boulanger, F. 2015, Proceedings IAU Symposium 316, Article ID IAU-15IAUS316-0210: Stellar Feedback From a Massive Super Star Cluster in the Antennae Merger Johnson, K. E., Leroy, A. K., Indebetouw, R., Brogan, C. L.,Whitmore, B. C., Hibbard, J., Sheth, K., \& Evans, A. S. 2015, ApJ, 806, 35

Schulz, A., Henkel, C., Muders, D., Mao, R. Q, Röllig, M., \& Mauersberger, R. 2007, A\& $A, 466$, 467

Teyssier, R., Chapon, D., \& Bournaud, F. 2010, ApJ, 720, 149

Ueda, J., Iono, D., Petitpas, G., Yun, M. S., Ho, P. T. P., Kawabe, R., Mao, R.-Q., Martín, S., Matsushita, S., Peck, A. B., Tamura, Y., Wang, J., Wang, Z., Wilson, C. D., \& Zhang, Q., 2012, ApJ, 745, 65

Weidner, C., Bonnell, I. A., \& Zinnecker, H. 2010, ApJ, 724, 1503

Whitmore, B. C., Brogan, C., Chandar, R., Evans, A., Hibbard, J., Johnson, K., Leroy, A., Privon, G., Remijan, A., \& Sheth, K. 2014, ApJ, 795, 156

Whitmore, B. C. \& Schweizer, F. 1995, AJ, 109, 960

Wilson, C., Scoville, N., Madden, S., \& Charmandaris, V. 2000, ApJ, 542, 120 